\title{
Noninvasive ventilation bi-pap basic use and modes
}

\begin{abstract}
While working in a good hospital setup according to my country standards I realize that few people are well aware of non invasive ventilation and its importance. So I am discussing non invasive BI-PAP ventilation and also describing basic modes.
\end{abstract}

Keywords: BI-PAP, non invasive ventilations, modes
Volume 3 Issue 3 - 2016

\author{
Sana Rashid \\ University of Health Sciences (GD-PGMI), Pakistan
}

Correspondence: Sana Rashid, University of Health Sciences (GD-PGMI), Lahore, Pakistan, Email sanarashidrt@gmail.com

Received: February 22, 2016| Published: May 23, 2016

Abbreviations: BI-PAP, bi level positive airway pressure; S, spontaneous; S/TD, spontaneous timed; T, timed; IPAP, inspiratory positive airway pressure; EPAP, expiratory positive airway pressure; $\mathrm{Ti}$, inspiratory time; $\mathrm{GB}$, guillian berry syndrome

\section{Introduction}

Non invasive ventilation is a type of mechanical ventilation in which we do not use invasive techniques like passing endotracheal tube. It is of two types C-PAP and Bi-PAP I am only discussing later. ${ }^{1}$ In Bi-PAP we give two levels of pressure IPAP and EPAP they are set after assessing patients condition and type of disease. IPAP is basically the positive airway pressure that we give in inspiration and EPAP is expiratory positive airway pressure that prevents our alveoli from collapsing and keep them open. ${ }^{2}$ BiPAP basic modes are $\mathrm{S}, \mathrm{St}$ and $\mathrm{T}$.

Spontaneous mode (S): In s mode all the breaths are patient triggered and cycled. Machine delivers pre set pressure during inspiration and expiration. No automatically delivered breath is inhaled. Pt initiate all the breaths and machine respond to inspiration and expiration.

Spontaneous timed mode (S/TD): In S/TD mode breaths are patient triggered and machine trigerred, patient cycled and machine cycled. We set backup breaths and also Ti. If patient do not initiate the breath during the set time then machine automatically delivers the breath.

Timed mode (T): In T mode machine does not rely on patient breath triggering effort. All the breaths are automatically delivered and set. So it is a machine cycled and machine triggered mode.

Bi-PAP ventilation a type of non invasive ventilation have a very important role in preventing patients from invasive ventilation its hazards and complications. ${ }^{3} \mathrm{Bi}$-PAP is always preferred over invasive ventilation and is a first step therapy in respiratory failures. It has a major role in acute respiratory failures. ${ }^{4}$ It is used in weaning pts from ventilation. It is also used in post-op patients. ${ }^{5}$ It is also used in neurological patients like muscular dystrophy, GB syndrome and motor neuron disease etc. ${ }^{6}$

\section{Conclusion}

All the modes of bi-pap should be understood and properly used. Awareness related to the use and modes of bi-pap should be spread. This will be beneficial for patients and home care providers.

\section{Acknowledgements}

None.

\section{Conflict of interest}

The author declares no conflict of interest.

\section{References}

1. J Masip. Heart failure reviews. 2007.

2. American Journal of Respiratory Medicine August. 2003;2(4):343-347.

3. Lightowler JV, Wedzicha JA, Elliott MW, et al. Non-invasive positive pressure ventilation to treat respiratory failure resulting from exacerbations of chronic obstructive pulmonary disease: Cochrane systematic review and meta-analysis. BMJ. 2003;326(7382):185.

4. Bourke SC, Tomlinson M, Williams TL, et al. Effects of non-invasive ventilation on survival and quality of life in patients with amyotrophic lateral sclerosis: a randomised controlled trial. Lancet Neurol. 2006;5(2):140-147.

5. Joris JL, Sottiaux TM, Chiche JD, et al. Effect of bi-level positive airway pressure (BiPAP) nasal ventilation on the postoperative pulmonary restrictive syndrome in obese patients undergoing gastroplasty. Chest. 1997;111(3):665-670.

6. Ward S, Chatwin M, Heather S, et al. Randomised controlled trial of non-invasive ventilation (NIV) for nocturnal hypoventilation in neuromuscular and chest wall disease patients with daytime normocapnia. Thorax. 2005;60(12):1019-1024. 